\title{
Measurement of Lateral Charge Diffusion in Thick, Fully Depleted, Back-illuminated CCDs
}

\author{
Armin Karcher, Christopher J. Bebek, Member, IEEE, William F. Kolbe, Dominic Maurath, \\ Valmiki Prasad, Michela Uslenghi, Martin Wagner
}

\begin{abstract}
Lateral charge diffusion in back-illuminated CCDs directly affects the point spread function (PSF) and spatial resolution of an imaging device. This can be of particular concern in thick, back-illuminated CCDs. We describe a technique of measuring this diffusion and present PSF measurements for an $800 \times 1100,15 \mu \mathrm{m}$ pixel, $280 \mu \mathrm{m}$ thick, back-illuminated, p-channel CCD that can be over-depleted. The PSF is measured over a wavelength range of $450 \mathrm{~nm}$ to $650 \mathrm{~nm}$ and at substrate bias voltages between $6 \mathrm{~V}$ and $80 \mathrm{~V}$.
\end{abstract}

\section{INTRODUCTION}

The spatial resolution of a CCD is determined by pixel size and lateral charge diffusion. The charge diffusion can be of concern in back-illuminated devices due to the distance between the point of charge generation and charge collection. Thick, back-illuminated devices generally have higher quantum efficiencies over a broader range of wavelengths, as well as less fringing at near-infrared wavelengths, making them useful for a variety of astronomy applications. But thick devices can have large lateral diffusion unless carefully designed. In most back-illuminated CCDs, self depletion typically occurs within a $\sim 10 \mu \mathrm{m}$ region of the front side leaving a significant non-depleted region at the backside and this results in a point spread function rms width comparable to the thickness of this field free region, typically of order $10 \mu \mathrm{m}$.

The Lawrence Berkeley National Laboratory (LBNL) CCD technology [1]-[3] allows control of lateral charge diffusion. The CCDs are fabricated on 200 to $300 \mu \mathrm{m}$ thick weaklydoped n-type substrates with donor densities of $\sim 10^{11} / \mathrm{cm}^{3}$ (>5 $\mathrm{k} \Omega-\mathrm{cm}$ ). The substrate can be fully depleted, even over depleted. The operation of the CCD including clocking and output source follower can be optimized for good charge

Manuscript received November 2003; revised May 2004.

This work was supported by the Director, Office of Science, of the U.S. Department of Energy under Contract No. DE AC03-76SF00098.

C. J. Bebek, A. Karcher, W. F. Kolbe, and V. Prasad are with the Lawrence Berkeley National Laboratory, Berkeley, CA 94720-8164 USA (telephone: 510486-6447, e-mail: cjbebek@lbl.gov).

D. Maurath and M. Wagner are with the Department of Sensorsystem Technology, Fachhochschule Karlsruhe - Hochschule für Technik, Karlsruhe, Germany.

M. Uslenghi is with the Instituto di Fisica Cosmica, Milan, Italy. transfer, well depth, and gain independent of the substrate bias voltage. When fully depleted, no field free region exists and charges are directed by an electric field during their entire drift time to the collection well. In this paper, we describe a technique of measuring the lateral charge diffusion and present experimental PSF results for an LBNL CCD.

Our long term goal is to populate the focal plane of a diffraction-limited telescope with $10^{9}$ pixels covering the wavelength range 0.35 to $1.0 \mu \mathrm{m}$. For the same angular coverage on the sky, a small-pixel-size CCD will require less instrumented area but the lateral charge diffusion must be commensurate with the pixel size so as not to significantly degrade the telescope PSF. To maintain good red response we want to maintain the CCD thickness at $200 \mu \mathrm{m}$ and still achieve an rms PSF of $4 \mu \mathrm{m}$. Since the authors are interested in photometry of point-like objects, we choose to use PSF as a measure of lateral charge diffusion impact rather than modulation transfer function.

\section{Measurement Principle And TeChniQuE}

We measure the charge profile of a point light source on the CCD to determine the PSF. Our measurement technique is based on the Foucault knife-edge technique. Instead of a physical knife edge, we select a grid of pixels and measure the variation of the total charge in the grid as the beam is scanned across a grid edge, a virtual knife edge. As shown in Fig. 2, the grid (integration region) is chosen such that initially the beam is completely contained within it. At the end of the scan, the beam lies outside the grid. The variation of the total charge in the integration region as a function of the beam position relative to the grid edge yields the charge profile in the scan direction as shown in the top of Fig. 3. The charge profile is one of two forms depending on the depletion regime, (1a) for under depletion where some fraction of the drift path is field free and (1b) for over depletion where the charges experience no field-free drift region [3], [4]

$$
\begin{aligned}
& Q(x)=a-\frac{(a-b)}{\pi \sigma / k} \int_{-\infty}^{x} 1 / \cosh \left(\frac{x^{\prime}-c}{\sigma / k}\right) d x^{\prime} \quad k=1.368 \\
& Q(x)=a-\frac{(a-b)}{\sqrt{2 \pi} \sigma} \int_{-\infty}^{x} \exp \left(\frac{-\left(x^{\prime}-c\right)^{2}}{2 \sigma^{2}}\right) d x^{\prime}
\end{aligned}
$$


where $a$ is the asymptotic charge value when the beam is fully contained in the integration-region, $b$ is the asymptotic value when the beam is fully outside the integration region, $c$ is the beam-center location where the integrated charge is half of $a-b$. Ideally, $b$ would be zero but it accommodates electronics offsets. $\sigma$ is the rms of the charge profile in the gaussian regime of (1b) and $k$ is defined so that

$$
\frac{1}{\pi \sigma / k} \int_{-\sigma}^{\sigma} 1 / \cosh \left(\frac{x^{\prime}}{\sigma / k}\right) d x^{\prime}=\frac{1}{\sqrt{2 \pi} \sigma} \int_{-\sigma}^{\sigma} \exp \left(\frac{-x^{\prime 2}}{2 \sigma^{2}}\right) d x^{\prime}
$$

The derivative of the integral charge profile contains the combined shapes of the source beam and the CCD response shown in the bottom of Fig. 3. The advantages of this technique over other approaches are independence of the pixel size and no scattering from a physical knife edge. The derivatives of (1a) or (1b) are fit to the data.

\section{EXPERIMENTAL APPARATUS}

A schematic of the experimental setup is shown in Fig. 4. The CCD is mounted in a liquid nitrogen cooled dewar that has been fitted with a thin, wide-band anti-reflection coated window and a mechanical shutter. The dewar is evacuated to $\sim 10^{-7}$ torr and the CCD is cooled to $133 \mathrm{~K}$. The temperature is regulated to an accuracy of about $1 \mathrm{~K}$.

A small diameter light source is needed to minimize its contribution to the charge profile in the CCD. An intense beam is needed to decrease the statistical uncertainties in the measurement. To achieve this, we use a GE 1493 tungsten bulb operated at $\sim 7 \mathrm{~W}$ behind a multi-lens collimator system. The collimated light beam passes through a filter wheel consisting of a set of Corion $70 \mathrm{~nm}$ bandwidth interference filters. The beam is focused using a convex lens onto an optical fiber that transmits the light to a pinhole projector. The pinhole projector is comprised of a $25.4 \mathrm{~mm}$ long brass tube that encloses a $10 \mu \mathrm{m}$ diameter pinhole and a 5x longworking-distance Mitutoyo objective. The latter has a working range between $450 \mathrm{~nm}$ and $650 \mathrm{~nm}$ and a depth of focus of $\sim 30 \mu \mathrm{m}$. The inner surface of the projector tube is threaded and blackened to reduce scattered light in the projected beam. Light-tight rubber bellows are placed between the projector and the dewar to prevent ambient light from reaching the CCD.

The pinhole projector is mounted onto an $\mathrm{x}-\mathrm{y}-\mathrm{z}$ translation stage, where the $x-y$ plane is carefully aligned to the CCD plane, with the $\mathrm{x}$-axis parallel and $\mathrm{y}$-axis perpendicular to the CCD rows. The dewar is kinematically mounted to an aluminum plate that, along with the projector translation stage, sits on a Newport isolation table.

Projector $\mathrm{x}-\mathrm{y}$ motions are implemented with stepper motors with step sizes of $0.4 \mu \mathrm{m}$. The position of the projector is read out using a non-contact linear optical encoder with an accuracy of about $0.1 \mu \mathrm{m}$. Movement in the z-direction is needed to focus the beam at the CCD surface and is performed manually using two micrometer screws, one for rough focus adjustment and the other for fine adjustment ( $1 \mu \mathrm{m} /$ division $)$.

A modified Astronomical Research Cameras Gen II controller is used to read out and control the operation of the CCD. A timing board generates the clock sequences and a clock driver board provides various programmable voltages for CCD operation. A video processor board filters, amplifies and digitizes the CCD output. The control of the shutter exposure time, projector $\mathrm{x}-\mathrm{y}$ motion, linear encoder operation, and temperature adjustment are performed using the controller utility board. Java-based controller software manages data-taking, while IDL-based software is used to process and analyze the data.

\section{EXPERIMENTAL PROCEDURE}

With the setup in Fig. 4, the charge profile is determined by moving the focused beam across the surface of the CCD in the $\mathrm{x}$ and $\mathrm{y}$ directions, corresponding to the rows and columns of the CCD, respectively. The beam is scanned across the CCD in 1.2 to $1.4 \mu \mathrm{m}$ steps, with larger step sizes used for lower substrate bias voltages. At each step, a 0.5-3 s exposure is taken. The shutter exposure time and lamp intensity are optimized to minimize statistical uncertainties while ensuring that there are no saturated pixels. Each scan covers a distance of 16-20 pixels, sufficient for measuring the charge profile at different points along the CCD. The data collection and analysis procedures are described below.

\section{A. Projector beam profile}

A modified setup of the apparatus in Fig. 4 is used to measure the pinhole projector beam profile. A physical knife edge and a photomultiplier tube (PMT) are placed in front of the dewar. The beam is focused on the knife edge and the total current in the PMT is measured as the pinhole projector is scanned across the knife edge. The variation of the PMT current with scan position is fitted to (1b) to extract the intrinsic beam width. The procedure was performed at the three wavelengths used in the lateral charge diffusion measurement. The beam width measurements are shown in Table I. The wavelength-averaged rms beam width, $\sigma_{\text {beam }}$, is $1.3 \pm 0.1 \mu \mathrm{m}$.

\section{B. z alignment - focusing}

To align the system, it is first necessary to focus the beam on the CCD. This is accomplished by moving the projector in the z-direction until the beam spot size on the CCD is minimized. The ratio of the charge in the pixel with the maximum charge to its neighboring pixels serves as a measure of beam focus. To focus the system properly, we apply a large 
substrate bias $(\sim 60 \mathrm{~V})$, thus depleting the substrate fully and reducing lateral charge diffusion. The perpendicularity of the projector axis to the CCD surface is checked by verifying that the beam remains focused during an $\mathrm{x}$ or $\mathrm{y}$ scan across the CCD.

\section{C. $x$-y alignment}

The $x-y$ alignment of the CCD and projector is determined by centering the beam on a pixel and then comparing the ratio of charges in the two rows or columns adjacent to the row or column containing the central pixel as the beam is scanned in the $\mathrm{x}$ or $\mathrm{y}$ direction. A variation in the ratio of charges with the scan position indicates a misalignment between the projector motion and the CCD. The substrate bias voltage is reduced to $\sim 40 \mathrm{~V}$ during these scans so that there is appreciable charge in the pixels surrounding the central pixel. The beam is scanned across $\sim 10$ pixels in $1.2 \mu \mathrm{m}$ steps and $3 \mathrm{~s}$ exposure images are acquired. After aligning the system using the $\mathrm{x}-\mathrm{y}-\mathrm{z}$ translation stage, we used a Monte Carlo technique to extract the beam position variation during the scan from the charge distribution variation. We find a typical $1 \mu \mathrm{m}$ linear y-drift per $100 \mu \mathrm{m}$ x motion and a $\pm 2.5 \mu \mathrm{m}$ oscillatory x-motion per $100 \mu \mathrm{m}$ y motion.

\section{Image offset level}

Before processing each image, the quality of each image is checked. Images without data-taking problems and cosmic rays are processed by subtracting an overall image offset and correcting for inter-pixel gain variations. We measure the average offset per image from a square annular region in the CCD that is illuminated with the beam. The rms uncertainty in determining the offset is included in the total charge measurement uncertainty in the integration region.

\section{E. Inter pixel gain variation}

After subtracting the offset from each image, we correct for inter-pixel gain variation. The inter-pixel response is measured by defocusing the beam such that it illuminates the region of the CCD that we are investigating. To reduce the effect of spatial variations in the unfocussed beam, the interpixel response is obtained by averaging a series of dithered and rotated images. We measure the pixel-to-pixel variations for three wavelengths $(450 \mathrm{~nm}, 550 \mathrm{~nm}$, and $650 \mathrm{~nm})$ and find them to be $\sim 5 \%$.

\section{F. Image normalization}

Fig. 5 shows that the total charge collected on the CCD varies by $\sim 5 \%$ during a scan. This is due to variations in the lamp-intensity and sub-pixel response. To correct for these variations, we normalize the total charge in the integration region to the total charge collected on the CCD for each image.

\section{EXPERIMENTAL RESULTS}

We measured the lateral charge diffusion for an $800 \times 1100$, $15 \mu \mathrm{m}$ pixel, $280 \mu \mathrm{m}$ thick, back-illuminated CCD that was fabricated at LBNL Micro Systems Lab. The PSF was determined at ten different substrate bias voltages between $6 \mathrm{~V}$ and $80 \mathrm{~V}$ and at three wavelengths: $450 \mathrm{~nm}, 550 \mathrm{~nm}$, and $650 \mathrm{~nm}$. Scans were performed in four directions, $\pm x$ and $\pm y$, over a $200 \times 200$-pixel region of the CCD. Typical measured charge profiles are shown in Fig. 6 for a few substrate bias voltages.

The charge profile width, $\sigma_{C C D}$, is defined such that $68 \%$ of the charge lies within $\pm \sigma_{C C D}$ of the beam center. To determine $\sigma_{C C D}$, we fit the rate of variation of the charge in the integration region with respect to the beam position as shown in Fig. 3. We fit (1a) for partially depleted measurements, $V_{\text {sub }}<15 \mathrm{~V}$, and (1b) for fully-depleted and over-depleted measurements.

We extract the PSF, $\sigma_{\text {diff }}$, from the relation

$$
\sigma_{\text {diff }}^{2}=\sigma_{C C D}^{2}-\sigma_{\text {beam }}^{2}
$$

Since $\sigma_{\text {beam }}$ is much smaller than the $\sigma_{C C D}$ results shown later, the PSF measurement is insensitive to the uncertainties in the intrinsic beam size.

$\sigma_{\text {diff }}$ was measured for different integration region sizes and positions. For each substrate voltage, wavelength, and scan, $\sigma_{\text {diff }}$ had a typical statistical uncertainty of $\sim 0.05 \mu \mathrm{m}$. For each substrate voltage and wavelength, the $\sigma_{\text {diff }}$ 's were distributed with an rms of $\sim 0.5 \mu \mathrm{m}$. This is included as one of the systematic uncertainties in the measurements.

The results of the PSF measurements are shown in Fig. 7 and are presented in Tables II, III, and IV. Errors quoted contain both statistical and systematic uncertainties. We do not observe any significant difference in $\sigma_{\text {diff }}$ at wavelengths between $450 \mathrm{~nm}$ and $650 \mathrm{~nm}$ nor between $\mathrm{x}$ and $\mathrm{y}$ scan directions. This is not surprising since the difference between the absorption lengths in silicon at $450 \mathrm{~nm}$ and $650 \mathrm{~nm}$ is only $2.7 \mu \mathrm{m}$. $\sigma_{\text {diff }}$ is seen to decrease rapidly with increasing substrate voltage in the partially-depleted region.

Fig. 7 shows a fit to the low voltage data based on the onedimensional analytical model in [3], equations (12) and (13),

$$
\sigma_{\text {diff }}=y_{D}-\sqrt{\frac{2 \varepsilon_{S i}}{q N_{D}}\left(V_{\text {sub }}-V_{j}\right)}
$$

where $y_{D}$ is the substrate thickness, $\varepsilon_{S i}$ is the permittivity of silicon, $q$ is the electron charge, $N_{D}$ is the donor density in the bulk substrate, $V_{\text {sub }}$ is the substrate bias voltage, and $V_{J}$ is the average channel potential. A fit yields $V_{J}=-1.4 \pm 0.5 \mathrm{~V}$ and $N_{D}=(3.1 \pm 0.2) \times 10^{11} / \mathrm{cm}^{3}$. This donor density 
corresponds to a bulk resistivity of $\sim 13.8 \mathrm{k} \Omega-\mathrm{cm}$, consistent with the bare silicon wafer resistivity of $\sim 10 \mathrm{k} \Omega-\mathrm{cm}$.

For the high voltage data, the theoretically $\sigma_{\text {diff }}$ approaches the constant-field result [2], [3]:

$$
\sigma_{\text {diff }}=\sqrt{\frac{2 \cdot k T}{q} \frac{y_{D}^{2}}{V_{\text {sub }}-V_{J}}}
$$

where $T$ is the absolute temperature and $k$ is the Boltzmann constant. The dashed line at high substrate voltages in Fig. 7 is (5) with $V_{J}$ determined by the low voltage fit. We note a consistent underestimate of the measured diffusion by this procedure.

We used the same scan images as those used to determine the charge profile to measure the accuracy of the $x-y$ position encoders. We measured the position of the center of each pixel and the distance between the pixel centers for substrate voltages greater than $44 \mathrm{~V}$. The center of each pixel is determined by fitting a Gaussian to the distribution of the fraction of total charge in the central pixel as a function of the encoder position. Fig. 8 shows the fractional charge in several adjacent pixels during a scan. The distribution of the distance between pixel centers is shown in Fig. 9. We observe that the mean pixel spacing is different for scans in the row and column directions and that there is indeed a trend in the pixel separation measurements for scans in the $\pm \mathrm{y}$ directions. The cause of this trend and the differences between scan directions is not understood at the moment. Combining row and column scans, the mean pixel separation is $15.0 \pm 0.5 \mu \mathrm{m}$. This $3.3 \%$ uncertainty in the encoder position measurement is the largest source of error in $\sigma_{\text {diff }}$.

\section{FUTURE WORK}

Future work includes repeating the above measurements for thinner CCDs, $200 \mu \mathrm{m}$ in particular, to verify the thickness scaling of the lateral diffusion predicted in (5). We will also measure smaller pixel sizes, in particular $10.5 \mu \mathrm{m}$, to verify that the virtual knife edge technique is indeed independent of pixel size. CCDs are in fabrication that can operate with a $150 \mathrm{~V}$ substrate bias. Again, this will provide more leverage to validate the voltage scaling of (5).

We are presently using the apparatus described here to measure lateral charge diffusion in other LBNL CCDs, varying in thickness and pixel size. With improvements in lamp stability and monitoring and eliminating the problem in the y positioning system, we plan to make use of the small projected beam profile to measure any intra-pixel variance that might exist in these CCDs.

\section{CONCLUSIONS}

We have successfully developed and applied a virtual knifeedge technique for measuring the lateral charge diffusion in a CCD. Using this technique, we measured the PSF of a $280 \mu \mathrm{m}$ thick, back-illuminated p-channel n-type overdepletable LBNL CCD. We demonstrated that the PSF width for this CCD varies from $89.9 \pm 3.0 \mu \mathrm{m}$ to $6.38 \pm 0.24 \mu \mathrm{m}$ as the substrate bias voltage increases from $6 \mathrm{~V}$ to $77 \mathrm{~V}$. Scaling according to (5), a $4 \mu \mathrm{m}$ rms diffusion should be achievable in a $200 \mu \mathrm{m}$ thick device operated at $80 \mathrm{~V}$.

\section{ACKNOWLEDGEMENTS}

We thank Steve Holland, Nick Palaio, and Guobin Wang of LBNL for manufacturing and providing the CCDs studied here. We also thank Steve Holland and Don Groom of LBNL for discussions on the theoretical underpinnings of the diffusion analysis.

\section{IX. .REFERENCES}

[1] S. E. Holland G. Goldhaber, D. E. Groom, W. W. Moses, C. R. Pennypacker, S. Perlmutter, N. W. Wang, R. J. Stover, and M. Wei,, "A 200 \$ltimes\$ 200 CCD image sensor fabricated on high-resistivity silicon," in IEDM Technical Digest, pp. 911-914, 1996.

[2] S. E. Holland, N. W. Wang, and W. W. Moses, "Development of low noise, back-side illuminated silicon photodiode arrays," IEEE Trans. Nucl. Sci., vol. 44, no. 3, pp. 443-447, Jun. 1997.

[3] S. E. Holland, D. E. Groom, N. P. Palaio, R. J. Stover, and M. Wei, "Fully Depleted, Back-Illuminated Charge-Coupled Devices Fabricated on Highresistivity Silicon,” IEEE Trans. Electron Devices, vol. 50, pp. 225-238, Jan. 2003.

[4] D. E. Groom, P. H. Eberhard, S. E. Holland, M. E. Levi, N. P. Palaio, S. Perlmutter, R. J. Stover, and M. Wei., "Point-spread function in depleted and partially depleted CCDs," in Proc. 4th ESO Workshop on Optical Detectors for Astronomy, Garching, Germany, 13-16 Sep. 1999. 
Fig. 1. At the top is shown a cross section of the CCD. A conventional three-phase, polysilicon gate structure is deposited on 200-300 $\mu$ m thick n-type, high-resistivity silicon. The channel implants are p-type. For back-illumination and backside electrical contact, a $2000 \AA$ in situ poly-doped window is deposited. The depletion voltage is applied to this contact. At the bottom, a MEDICI simulation of the electric field near the collection surface of the CCD is shown. The bias voltage is $40 \mathrm{~V}$. Three clock gates are shown near $y=0$ with the middle gate in collection phase. Moving away from the pixel region, positive $y$, the electric field is seen to be spatially uniform and of approximately linear. This linear region extends to the backside of the CCD, leaving no field-free region.

Fig. 2: Schematic for virtual knife-edge technique.

Fig. 3: Top: Variation of total charge (normalized) in integration region versus scan position. The smooth curve shows a fit to an error function. Bottom: Derivative of total charge (normalized) variation in integration region versus scan position. The smooth curve shows a fit to a gaussian. The total charge in the integration region is normalized to the total charge read out on the CCD.

Fig. 4: Experimental setup used for lateral charge diffusion measurement.

Fig. 5: Variation of total charge collected on CCD versus scan position. The variation observed may be caused by a temporal fluctuation of the lamp intensity and/or intra-pixel response variations.

Fig. 6: Charge profiles on CCD at substrate voltages of $5.8 \mathrm{~V}, 12.1 \mathrm{~V}, 61.5 \mathrm{~V}$, and $76.8 \mathrm{~V}$.

Fig. 7: Left: Variation of PSF $\left(\sigma_{\text {diff }}\right)$ with substrate bias voltage $\left(V_{\text {sub }}\right)$ at $450 \mathrm{~nm}, 550 \mathrm{~nm}$, and $650 \mathrm{~nm}$ in the x-(row) direction. Right: Variation of PSF

$\left(\sigma_{\text {diff }}\right)$ with substrate bias voltage $\left(V_{\text {sub }}\right)$ at $650 \mathrm{~nm}$ in the $\mathrm{x}$ (row) and y (column) directions. The dashed curves indicate the theoretical fit based on the onedimensional analytical model discussed in [3]. The error bars in both plots include both statistical and systematic uncertainties.

Fig. 8: Variation of the charge fraction in the central pixel versus encoder position for adjacent pixels.

Fig. 9: Variation of fraction of charge in central pixel versus encoder position. Left is for a row scan; right is for a column scan. The deviation of the average position from $15 \mu \mathrm{m}$ can be attributed to position encoder calibrations. The distorted distribution for the column scan is under investigation.

Table I: Intrinsic beam width measurements.

Table II: Measurements of $\sigma_{\text {diff }}$ at $450 \mathrm{~nm}$.

Table III: Measurements of $\sigma_{\text {diff }}$ at $550 \mathrm{~nm}$.

Table IV: Measurements of $\sigma_{\text {diff }}$ at $650 \mathrm{~nm}$. 
Table I: Intrinsic beam width measurements.

\begin{tabular}{|c|c|}
\hline Wavelength $(\mathrm{nm})$ & $\sigma_{\text {beam }}(\mu \mathrm{m})$ \\
\hline 450 & $1.26 \pm 0.10$ \\
550 & $1.19 \pm 0.10$ \\
650 & $1.39 \pm 0.10$ \\
\hline
\end{tabular}




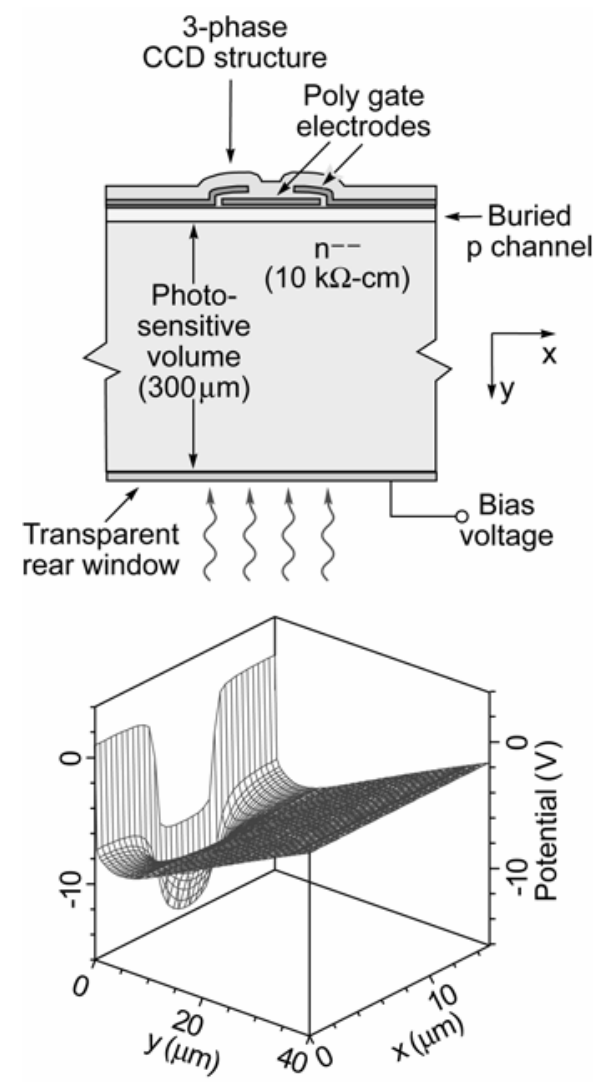

Fig. 1. At the top is shown a cross section of the CCD. A conventional three-phase, polysilicon gate structure is deposited on 200-300 $\mu \mathrm{m}$ thick n-type, high-resistivity silicon. The channel implants are p-type. For back-illumination and backside electrical contact, a 2000 $\AA$ in situ poly-doped window is deposited. The depletion voltage is applied to this contact. At the bottom, a MEDICI simulation of the electric field near the collection surface of the CCD is shown. The bias voltage is $40 \mathrm{~V}$. Three clock gates are shown near $\mathrm{y}=0$ with the middle gate in collection phase. Moving away from the pixel region, positive $y$, the electric field is seen to be spatially uniform and of approximately linear. This linear region extends to the backside of the CCD, leaving no field-free region. 


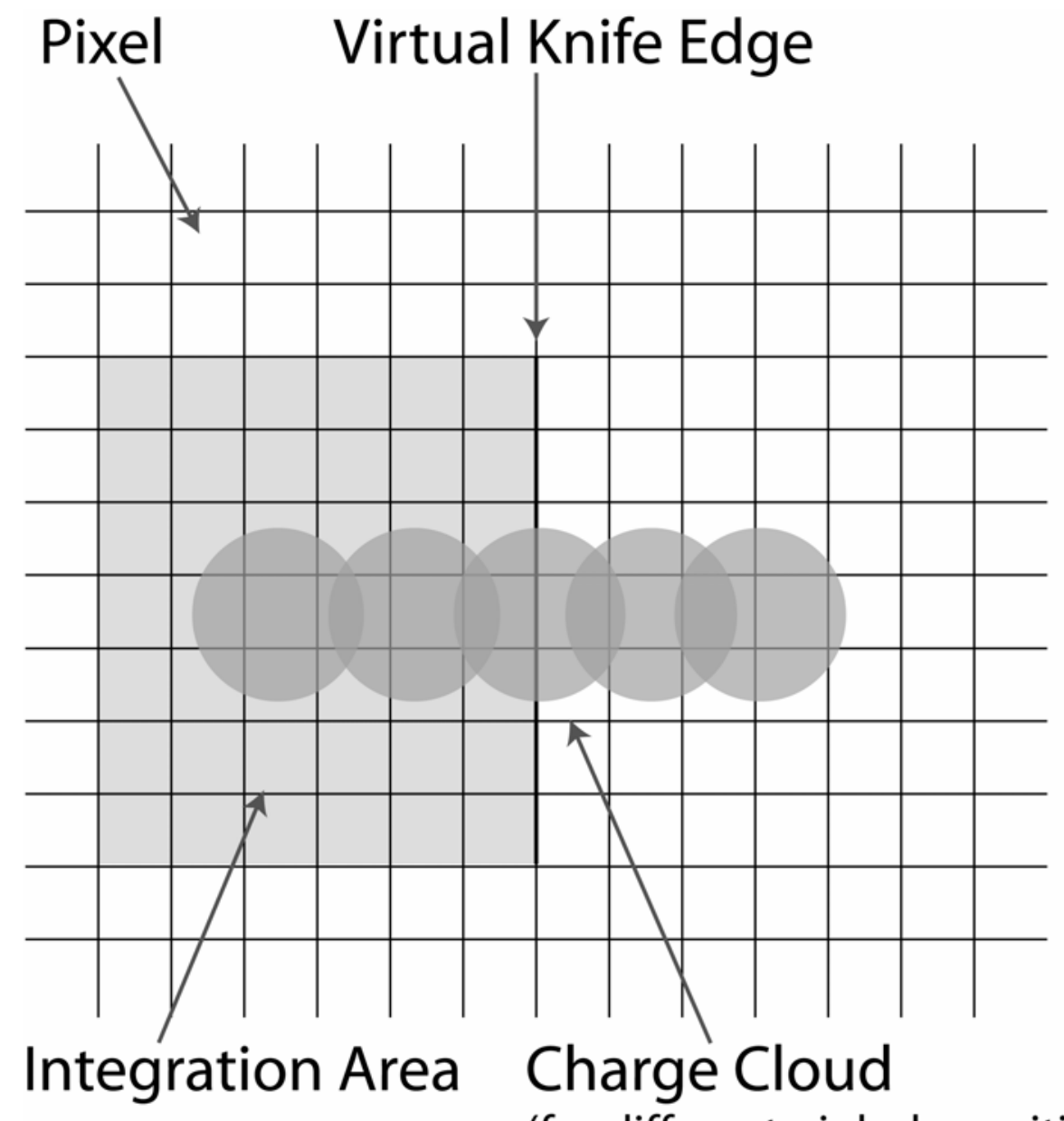

(for different pinhole positions)

Fig. 2: Schematic for virtual knife-edge technique. 

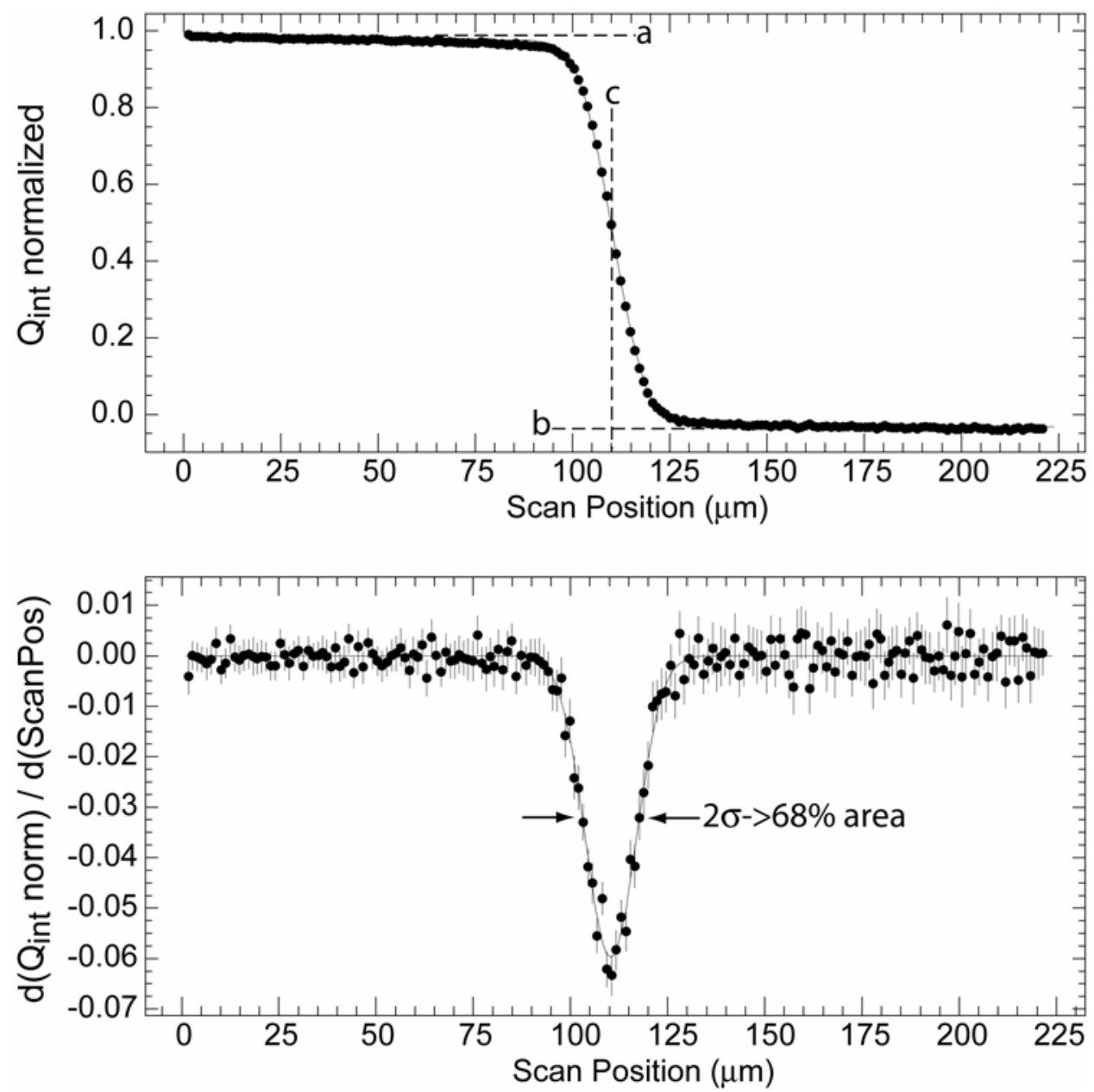

Fig. 3: Top: Variation of total charge (normalized) in integration region versus scan position. The smooth curve shows a fit to an error function. Bottom: Derivative of total charge (normalized) variation in integration region versus scan position. The smooth curve shows a fit to a gaussian. The total charge in the integration region is normalized to the total charge read out on the CCD. 


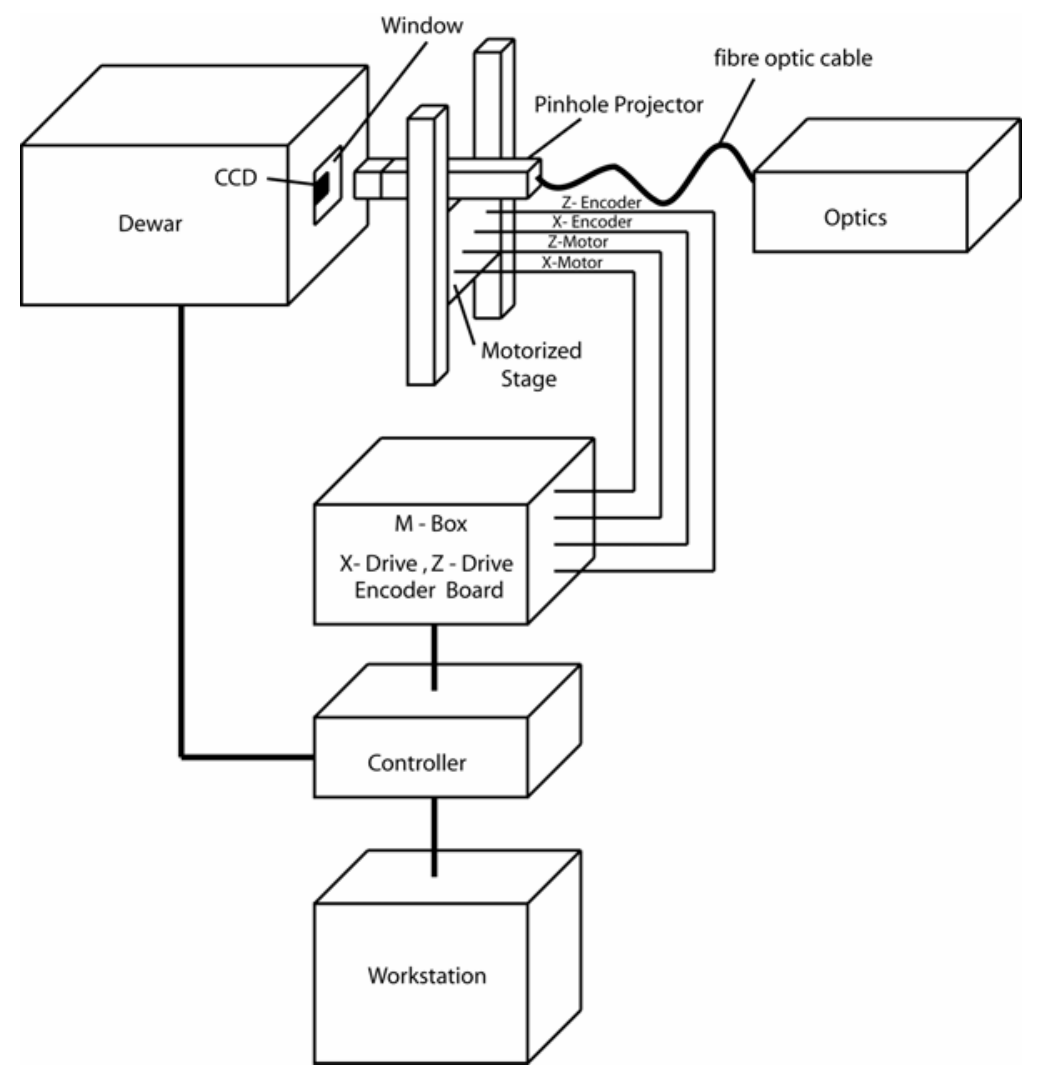

Fig. 4: Experimental setup used for lateral charge diffusion measurement. 


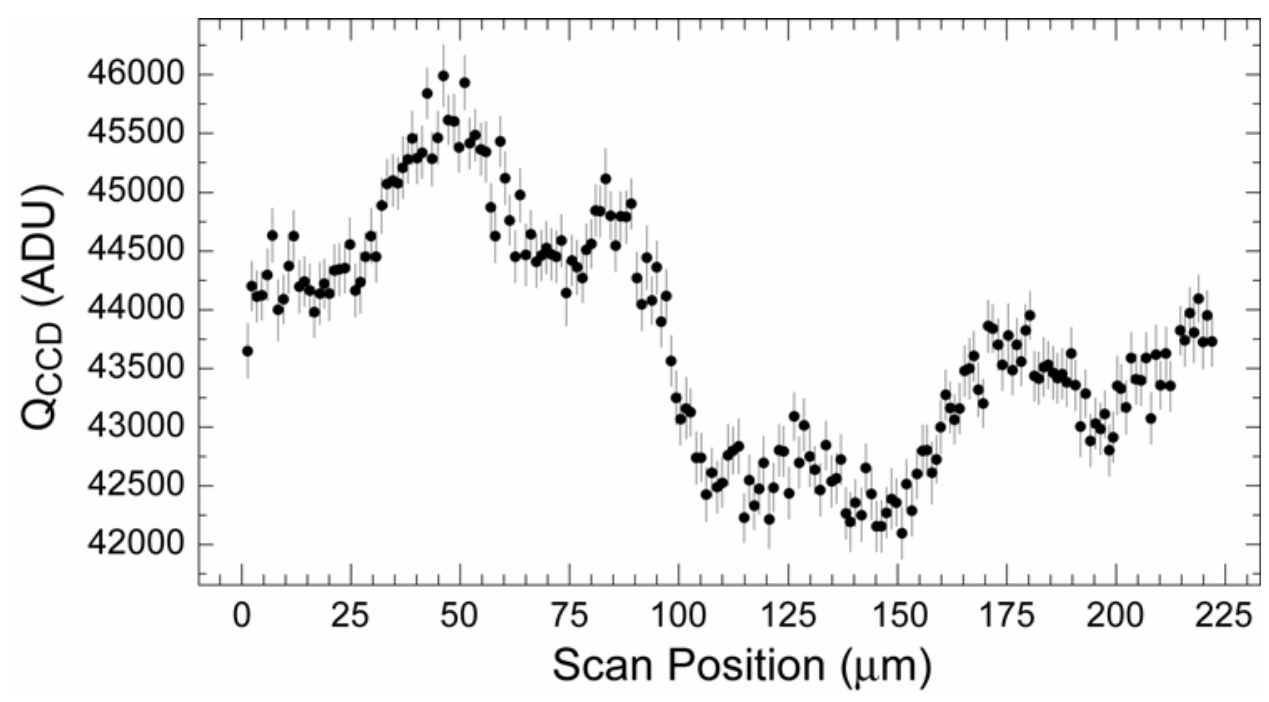

Fig. 5: Variation of total charge collected on CCD versus scan position. The variation observed may be caused by a temporal fluctuation of the lamp intensity and/or intra-pixel response variations. 

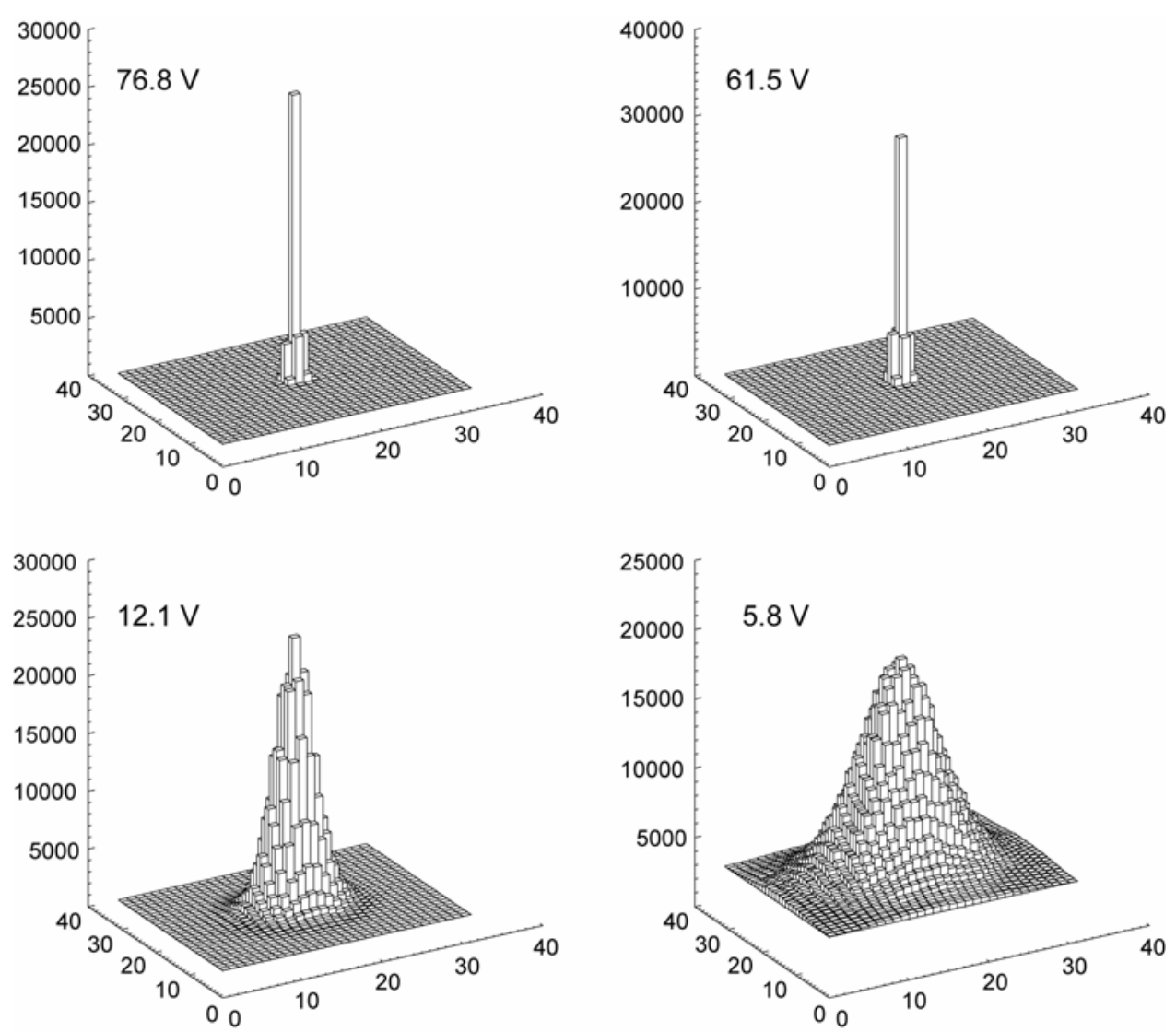

Fig. 6: Charge profiles on CCD at substrate voltages of $5.8 \mathrm{~V}, 12.1 \mathrm{~V}, 61.5 \mathrm{~V}$, and $76.8 \mathrm{~V}$. 

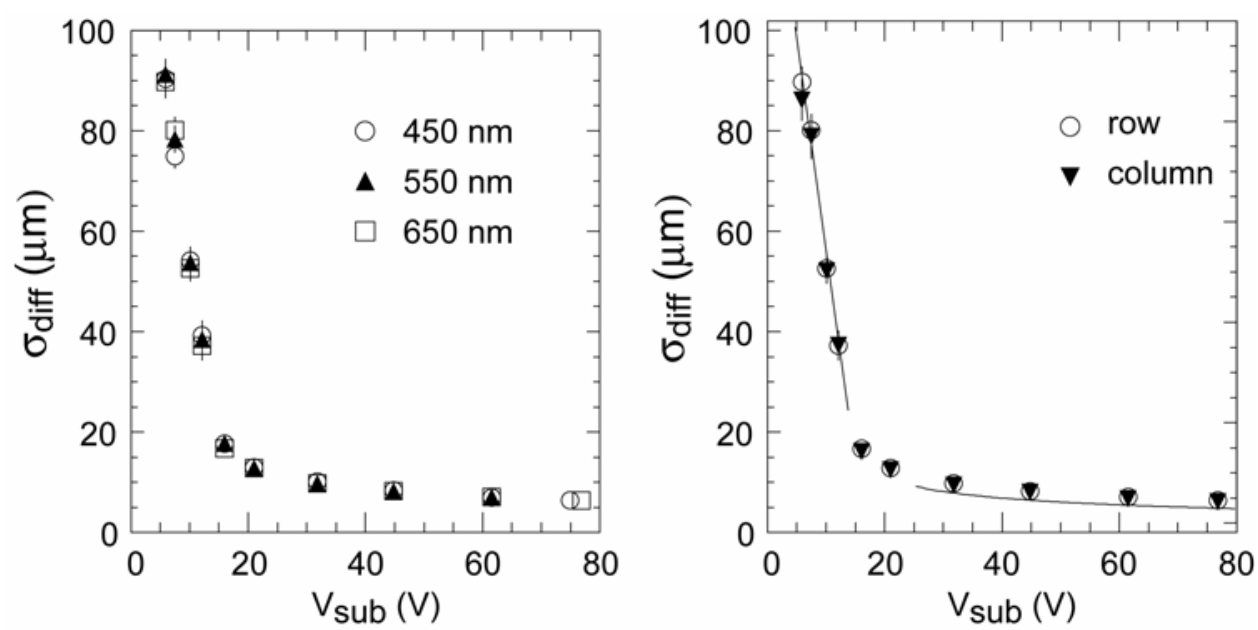

Fig. 7: Left: Variation of PSF ( $\left.\sigma_{\text {diff }}\right)$ with substrate bias voltage $\left(\mathrm{V}_{\text {sub }}\right)$ at $450 \mathrm{~nm}, 550 \mathrm{~nm}$, and $650 \mathrm{~nm}$ in the $\mathrm{x}$-(row) direction. Right: Variation of PSF $\left(\sigma_{\text {diff }}\right)$ with substrate bias voltage $\left(\mathrm{V}_{\text {sub }}\right)$ at $650 \mathrm{~nm}$ in the $\mathrm{x}$ (row) and $\mathrm{y}$ (column) directions. The dashed curves indicate the theoretical fit based on the one-dimensional analytical model discussed in [3]. The error bars in both plots include both statistical and systematic uncertainties. 


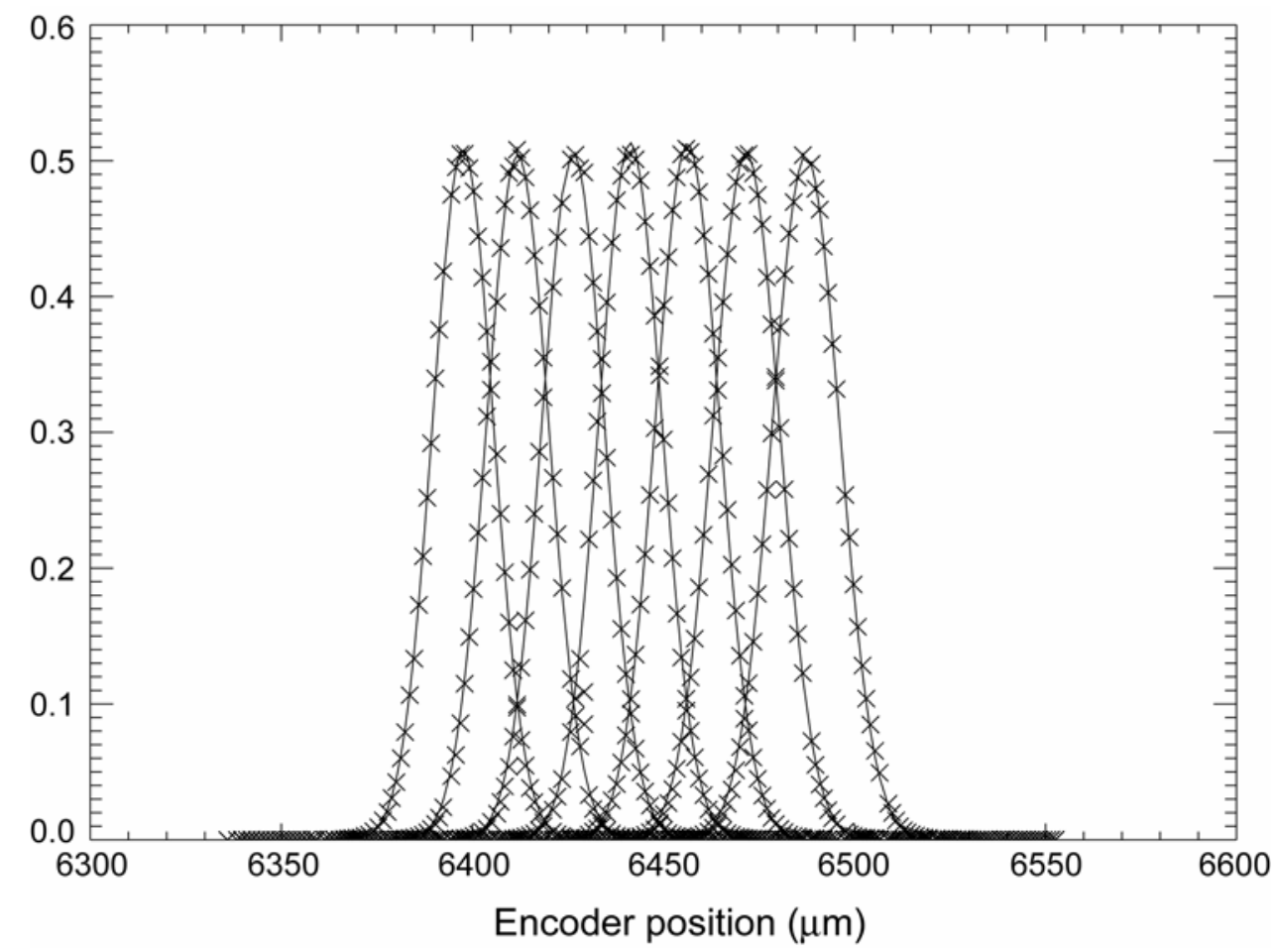

Fig. 8: Variation of the charge fraction in the central pixel versus encoder position for adjacent pixels. 

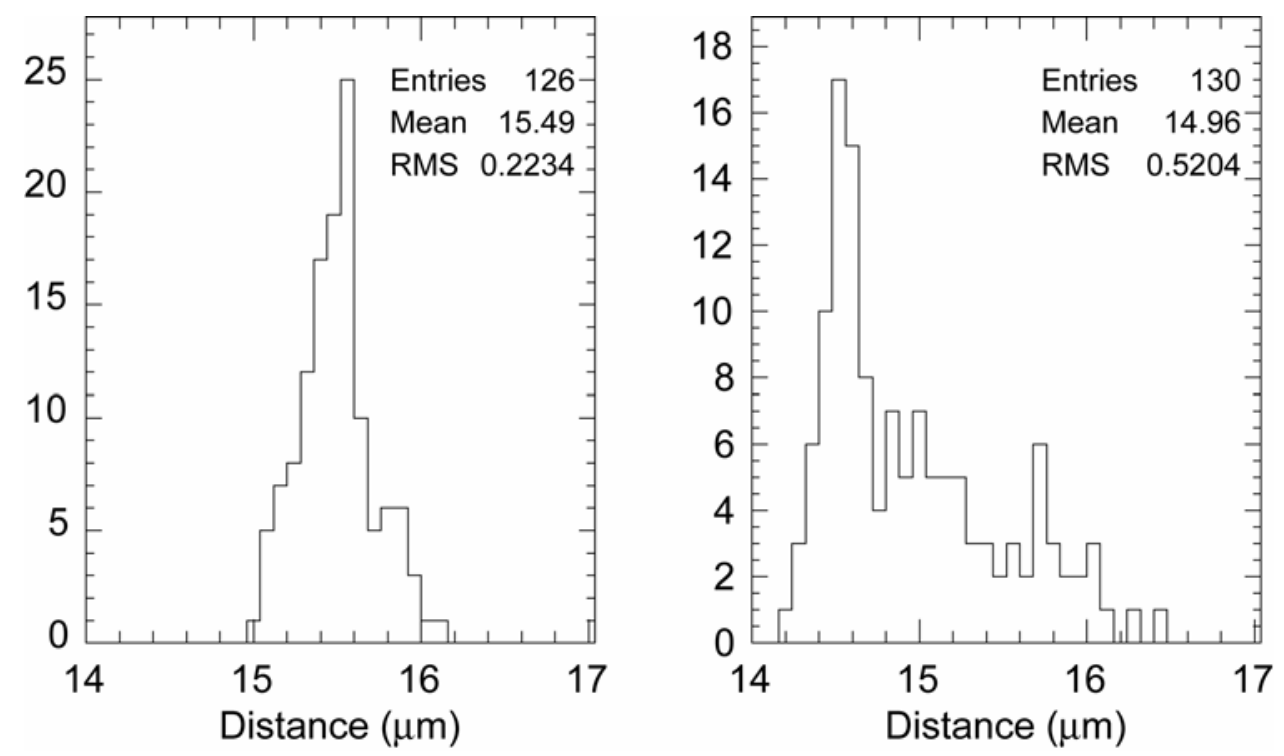

Fig. 9: Variation of fraction of charge in central pixel versus encoder position. Left is for a row scan; right is for a column scan. The deviation of the average position from $15 \mu \mathrm{m}$ can be attributed to position encoder calibrations. The distorted distribution for the column scan is under investigation. 
Table II: Measurements of $\sigma_{\text {diff }}$ at $450 \mathrm{~nm}$.

\begin{tabular}{|r|c|c|}
\hline & \multicolumn{2}{|c|}{$\sigma_{\text {diff }}(\mu \mathrm{m})$} \\
$\mathrm{V}_{\text {sub }}$ & $|\mathrm{x}|$ scan & $|\mathrm{y}|$ scan \\
\hline 5.9 & $90.8 \pm 3.9$ & $85.9 \pm 3.4$ \\
7.5 & $75.3 \pm 2.5$ & $73.7 \pm 2.5$ \\
10.1 & $54.4 \pm 2.8$ & $54.5 \pm 2.6$ \\
12.1 & $39.5 \pm 3.0$ & $36.8 \pm 1.6$ \\
16.0 & $17.7 \pm 0.8$ & $17.2 \pm 1.0$ \\
21.0 & $13.0 \pm 0.5$ & $12.8 \pm 0.8$ \\
31.8 & $10.1 \pm 0.4$ & $9.8 \pm 0.6$ \\
44.8 & $8.4 \pm 0.4$ & $8.2 \pm 0.5$ \\
61.5 & $7.0 \pm 0.3$ & $6.7 \pm 0.5$ \\
74.9 & $6.4 \pm 0.3$ & $6.3 \pm 0.4$ \\
\hline
\end{tabular}


Table III: Measurements of $\sigma_{\text {diff }}$ at $550 \mathrm{~nm}$.

\begin{tabular}{|r|c|c|}
\hline & \multicolumn{2}{|c|}{$\sigma_{\text {diff }}(\mu \mathrm{m})$} \\
$\mathrm{V}_{\text {sub }}$ & $|\mathrm{x}|$ scan & $|\mathrm{y}|$ scan \\
\hline 5.9 & $91.8 \pm 3.1$ & $90.1 \pm 3.0$ \\
7.5 & $78.8 \pm 2.7$ & $76.8 \pm 2.6$ \\
10.1 & $54.1 \pm 3.0$ & $54.5 \pm 2.4$ \\
12.1 & $38.7 \pm 3.0$ & $36.2 \pm 1.5$ \\
16.0 & $17.8 \pm 0.9$ & $17.0 \pm 0.9$ \\
21.0 & $12.8 \pm 0.5$ & $12.3 \pm 0.6$ \\
31.8 & $9.8 \pm 0.5$ & $9.5 \pm 0.5$ \\
44.8 & $8.3 \pm 0.3$ & $8.0 \pm 0.5$ \\
61.5 & $7.0 \pm 0.3$ & $6.8 \pm 0.4$ \\
78.8 & $6.6 \pm 0.3$ & $6.2 \pm 0.4$ \\
\hline
\end{tabular}


Table IV: Measurements of $\sigma_{\text {diff }}$ at $650 \mathrm{~nm}$.

\begin{tabular}{|r|c|c|}
\hline & \multicolumn{2}{|c|}{$\sigma_{\text {diff }}(\mu \mathrm{m})$} \\
$\mathrm{V}_{\text {sub }}$ & $|\mathrm{x}|$ scan & $|\mathrm{y}|$ scan \\
\hline 5.9 & $90.3 \pm 3.0$ & $86.6 \pm 4.2$ \\
7.5 & $80.6 \pm 2.7$ & $79.4 \pm 4.5$ \\
10.1 & $52.9 \pm 2.5$ & $52.4 \pm 2.6$ \\
12.1 & $37.4 \pm 3.0$ & $37.5 \pm 2.5$ \\
16.0 & $16.8 \pm 0.6$ & $16.1 \pm 0.7$ \\
21.0 & $12.8 \pm 0.6$ & $12.5 \pm 0.7$ \\
31.8 & $9.8 \pm 0.5$ & $9.4 \pm 0.5$ \\
44.8 & $8.2 \pm 0.5$ & $8.0 \pm 0.5$ \\
61.5 & $7.1 \pm 0.3$ & $6.8 \pm 0.4$ \\
76.8 & $6.4 \pm 0.3$ & $6.1 \pm 0.4$ \\
\hline
\end{tabular}

